CASE REPORT

\author{
T.A. Stark \\ A.M. McKinney \\ C.S. Palmer \\ R.H. Maisel \\ C.L. Truwit
}

\section{Dilation of the Subarachnoid Spaces Surrounding the Cranial Nerves with Petrous Apex Cephaloceles in Usher Syndrome}

\begin{abstract}
SUMMARY: Limited studies of brain MR imaging findings in Usher syndrome have reported atrophy with enlarged subarachnoid spaces. However, the specific appearance of the subarachnoid spaces surrounding the cranial nerves has not yet been described. Herein we describe the skull base MR imaging findings in an adult with Usher syndrome. Multiple cranial nerve exits were enlarged to the point of causing cephaloceles with bony remodeling. A combination of uncommon findings in this rare disorder raises the question of an etiologic association.
\end{abstract}

U sher syndrome, also called Graefe-Usher syndrome, is a rare, congenital, autosomal recessive disorder characterized by retinitis pigmentosa and sensorineural hearing loss, as first described by Von Graefe in 1858. ${ }^{1,2}$ In the United States, the prevalence is estimated at 4.4 per $100,000 .{ }^{1}$ Usher syndrome stems from a variety of genetic mutations for which a common mechanism is not well understood. ${ }^{3}$ This syndrome is divided into 3 clinical subtypes on the basis of the temporal course and the extent of deafness: type I patients exhibit profound hearing loss and lack of vestibular function; type II patients have moderate to severe hearing loss with preserved vestibular function; and type III patients have milder progressive hearing loss, with approximately half having vestibular deterioration. ${ }^{3}$ The CT and MR imaging literature describing Usher syndrome is sparse but describes diffuse brain atrophy and enlargement of the subarachnoid spaces with diminished cerebral and posterior fossa tissue volumes. ${ }^{4-6}$ There has not yet been a description of whether the subarachnoid space enlargement involves the transit points of the cranial nerves along the skull base.

A petrous apex cephalocele (PAC) is an uncommon lesion consisting of congenital or acquired herniation of the contents from the Meckel cave, which may cause remodeling of the adjacent petrous temporal bone. ${ }^{7}$ On pathologic examination, these have been defined as either meningoceles or arachnoid cysts. $^{7}$ These lesions are usually incidental and unilateral but rarely may be symptomatic.

Here we report the case of an adult with type I Usher syndrome, in whom MR imaging demonstrated diffuse dilation of the subarachnoid spaces extending along the cranial nerves to the point of causing bony remodeling from PACs. The presence of these uncommon findings in a patient with this rare disorder raises the question of an etiologic association.

\section{Case Report}

A 58-year-old man with type I Usher syndrome (per ophthalmologic diagnosis) and baseline ocular blindness and sensorineural hearing loss presented initially with temporal pain underlying the right tem-

Received June 29, 2008; accepted after revision July 16

From the Department of Radiology, University of Minnesota Medical School \& Hennepin County Medical Center, Minneapolis, Minn.

Please address correspondence to Alexander McKinney, MD, Hennepin County Medical Center, Department of Radiology, 701 Park Ave South, Minneapolis, MN 55415; e-mail: mckinrad@umn.edu

DOI 10.3174/ajnr.A1283 poromandibular joint. An MR imaging examination was performed on a $3 \mathrm{~T}$ scanner to exclude an intracranial cause, consisting of axial fast spin-echo (FSE) T2-weighted images (T2WI), diffusion, and fluid-attenuated inversion recovery images through the whole brain (5-mm thickness), with thinner 3D FSE T2WI images through the skull base $(0.6 \mathrm{~mm})$, as well as precontrast and postcontrast axial and coronal T1-weighted images of the skull base (2-mm thickness) with fat suppression.

On MR imaging, there were diffusely enlarged subarachnoid spaces surrounding the exits of cranial nerves (CN) II-XII, being most prominent along the oculomotor cisterns (CN III), Meckel caves (CN $\mathrm{V}$ ), and within the jugular foramina (CN X; Fig $1 A-F)$. The findings were bilateral and relatively symmetric, with the exception of enlargement of the Dorello canal unilaterally surrounding CN VI. These enlarged spaces followed the signal intensity of CSF on all sequences. The most prominent enlarged spaces were 3 - $\mathrm{cm}$-sized lesions of the petrous apices that were considered to be PACs because of their having CSF intensity that was contiguous with CSF within the Meckel cave. There was also moderate cerebral volume loss. There was no abnormal contrast enhancement of the brain parenchyma or meninges. A temporal bone CT confirmed the enlarged foramina and the petrous apex remodeling and showed a mildly enlarged bony sellar contour (Fig 2). An MR imaging examination 1 year previously was obtained for comparison and showed an identical appearance and size of the PACs.

The patient's symptoms were thought related to the temporomandibular joint after they resolved with nonsurgical therapy. A neuro-otologic consultation was then obtained to evaluate the petrous apex lesions; the neuro-otologist elected to observe these lesions. Nearly 2 years after presentation, the patient continues to be followed by otolaryngology should his symptoms worsen. No additional symptoms or cranial neuropathies have developed beyond his baseline blindness and sensorineural hearing loss.

\section{Discussion}

Although the underlying causes of Usher syndrome are not entirely clear, the best understood and most commonly accepted molecular mechanism in type I Usher syndrome involves the myosin VIIa protein., ${ }^{2,3}$ This protein encodes another unconventional myosin protein localized in the connecting cilium of photoreceptor cells in the retinal pigment epithelium and inner ear stereocilia. ${ }^{3}$ Reports of patients with type I Usher syndrome having bronchiectasis, chronic sinusitis, and primary ciliary aplasia suggest that the effects of these mutations are not limited to the inner ear and retina.,

PACs are thought to arise from increases in intracranial 

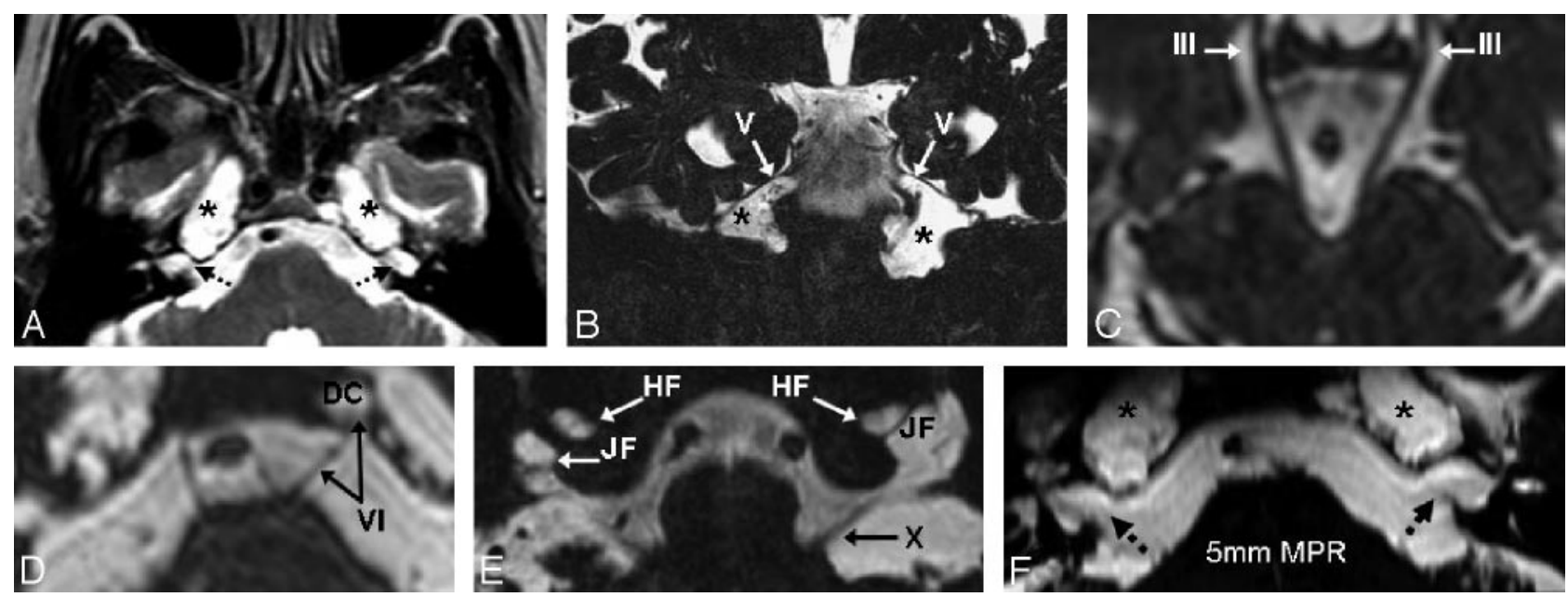

Fig 1. On MR imaging, the bilateral PACs were bright on T2WI (A) but not on diffusion or fluid-attenuated inversion recovery (not shown). Thin, 0.6-mm 3D FSE T2WI (B-E) demonstrated a communication with Meckel caves (asterisks) in Figs $1 A, 1 B$, and $1 F$ and depicted dilated subarachnoid spaces surrounding the cranial nerves. Shown are the oculomotor cistern (III in Fig $1 C$, Dorello canal in Fig $1 D(V I, D C)$, Meckel caves in Figs $1 A, 1 B$, and $1 F(V$ and asterisks), and the jugular (X, JA) and hypoglossal foramina (XII, HF) in Fig $1 E$. There are 5-mm-thick reconstructions from the 3D FSE T2WI that illustrate the PACs and the adjacent enlarged internal auditory canals (Fig 1 F). The prominent internal auditory canals in Figs $1 A$ and $1 F(d a s h e d$ arrows) did not communicate with the PACs.

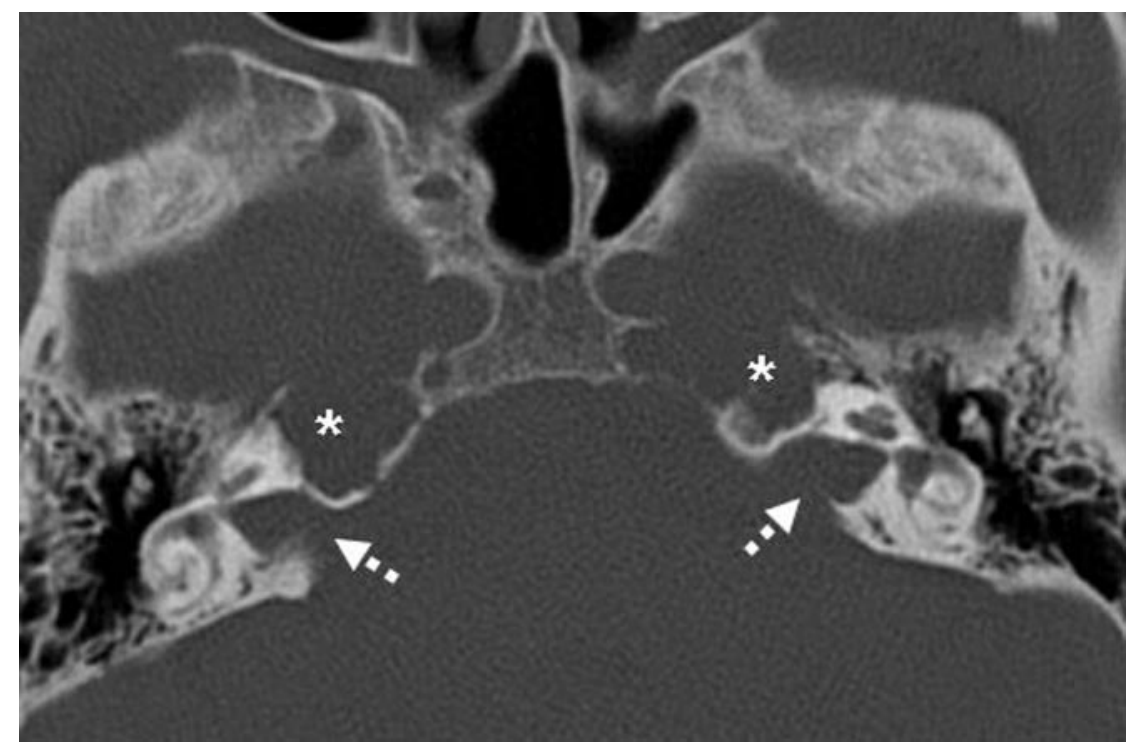

Fig 2. A CT scan was obtained after MR imaging for confirmation of the PACs and better characterization of the extent of bony remodeling of the petrous apices (asterisks). Note the enlarged internal auditory canals as well (dashed white arrows).

pressure, whether transient or sustained. ${ }^{10,11}$ Only 20 to 30 cases have been reported, 3 being bilateral, but their frequency is difficult to determine because these may have been underreported or listed under alternate entities such as meningoceles or arachnoid cysts, or even mistakenly identified as other common lesions of the petrous apex. ${ }^{10,11}$ In our patient, MR imaging ultimately excluded other lesions in the differential diagnosis of petrous apex masses, such as cholesterol granulomas (not T1-bright), epidermoid cysts (lack of decreased diffusion), bilateral glomus tumors or schwannomas (no enhancement), and petrous apicitis or effusions (no enhancement or mastoiditis).

The cause of the cystic dilations of the subarachnoid spaces in our patient is unclear, and the mechanism of formation of the PACs is unknown; however, these "cephaloceles" could result from a model similar to that proposed for arachnoid cyst formation. At approximately the 15th gestational week, the roof of the fourth ventricle opens into the cisterna magna via the foramina of Magendie and Luschka, and the CSF passing into the cisternal mesenchyme can result in arachnoid membrane splitting; this splitting may eventually lead to the formation of a CSF-containing cyst. ${ }^{1-14}$ Hence, in our patient, the PACs could result from a similar process occurring focally through the Meckel cave and the porus trigeminus as well as through the other pori associated with the other cranial nerve exits, resulting in the appearance of multiple cephaloceles. ${ }^{11}$ Also it has been postulated that intracranial hypertension or abnormal CSF pulsation can contribute to PAC enlargement. ${ }^{10,11}$

It is interesting to note that changes in intracranial pressure leading to hydrocephalus is a phenomenon that has been cited to occur in an emerging class of disorders of ciliary function, which includes Kartagener syndrome, Meckel-Gruber syndrome, Joubert syndrome, and other rare cilia dysfunctions, 
some of which may be associated with cephaloceles. ${ }^{15,16}$ Indeed, recent evidence from an animal model has demonstrated that in the ependymal cell layer, dysfunction of the cilia can cause aberrant ependymal flow and resultant closure of the cerebral aqueduct with subsequent hydrocephalus. ${ }^{16}$ From a theoretic standpoint, such dysfunction could have occurred to some degree in our patient, contributing to a low-level hydrocephalus and cephalocele formation. We note that our patient lacked the typical findings of acute hydrocephalus, such as enlarged temporal horns of the lateral ventricles, enlarged anterior recesses of the third ventricle, periependymal T2 hyperintensity, or enlarged ventricles relative to the cerebral sulci. On the other hand, a chronic, low-level hydrocephalus or other abnormality of CSF transmission or pulsation could not be entirely excluded, particularly given the mild bony sellar enlargement.

Another possibility is that the dilated subarachnoid spaces, the multiple cephaloceles, and the cerebral volume loss were separate, isolated findings. However, this is unlikely because PACs are quite uncommon and are especially rare to be bilateral, particularly in the setting of diffusely prominent subarachnoid spaces surrounding the cranial nerves. This combination of rare findings raises the question of a common, underlying mechanism related to abnormal CSF flow or pulsation. Although our patient did not exhibit any clear clinical findings of hydrocephalus, these signs can be nonspecific in states of chronically elevated intracranial pressure.

In the available literature on Usher syndrome, we have not found descriptions of PACs, nor have we found reports of enlarged subarachnoid spaces surrounding cranial nerve exits to the point of causing scalloping and remodeling of the skull base. The sparse literature describing the histopathologic findings in Usher syndrome focuses primarily on atrophic changes in the organ of Corti and ciliary aplasia. ${ }^{8,17}$ It is unfortunate that most reports regarding MR imaging findings in this syndrome have small patient sample sizes without long-term sur- veillance and have described nonspecific findings including brain atrophy and enlarged subarachnoid spaces, without mention of involvement of the cranial nerve exit sites or descriptions using thin-section MR imaging of the skull base. ${ }^{4-6}$

\section{References}

1. Boughman J, Vernon M, Shaver K. Usher syndrome: definition and estimate of prevalence from two high-risk populations. J Chronic Dis 1983;36:595-603

2. Riazuddin S, Nazli S, Ahmed ZM, et al. Mutation spectrum of MYO7a and evaluation of a novel nonsyndromic deafness DFNB2 allele with residual function. Hum Mutat 2008;29:502-11

3. Williams D. Usher syndrome: animal models, retinal function of usher proteins, and prospects for gene therapy. Vision Res 2008;48:433-41

4. Bloom T, Fishman G, Mafee M. Usher syndrome: CNS defects determined by computed tomography. Retina 1983;3:108-13

5. Tamayo M, Maldonado C, Plaza S, et al. Neuroradiology and clinical aspects of Usher syndrome. Clin Genet 1996;50:126-32

6. Schaefer G, Bodensteiner J, Thompson J, et al. Volumetric neuroimaging in Usher syndrome: evidence of global involvement. Am J Med Genet 1998;79:1-4

7. Moore K, Fischbein N, Harnsberger $\mathrm{H}$, et al. Petrous apex cephaloceles. AJNR Am J Neuroradiology 2001;22:1867-71

8. Tosi G, de Santi M, Pradal U, et al. Usher syndrome type 1 associated with primary ciliary aplasia. Arch Ophthalmol 2003;121:407-08

9. Bonneau D, Raymond F, Kremer C, et al. Usher syndrome type I associated with bronchiectasis and immotile nasal cilia in two brothers. J Med Genet 1993;30:253-54

10. Alorainy I. Petrous apex cephalocele and empty sella: is there a relation? Eur J Radiol 2007;62:378-84

11. Isaacson B, Coker N, Vrabec J, et al. Invasive cerebrospinal fluid cysts and cephaloceles of the petrous apex. Otol Neurotol 2006;27:1131-41

12. Abbott R. The endoscopic management of arachnoidal cysts. Neurosurg Clin N Am 2004;15:9-17

13. Starkman SP, Brown TC, Linell EA. Cerebral arachnoid cysts. J Neuropathol Exp Neurol 1958;17:484-500

14. Cristobal R, Oghalai JS. Peripetrosal arachnoid cysts. Curr Opin Otolaryngol Head Neck Surg 2007;15:323-29

15. Badano JL, Mitsuma N, Beales PL, et al. The ciliopathies: an emerging class of human genetic disorders. Annu Rev Genomics Hum Genet 2006;7:125-48

16. Ibañex-Tallon I, Gorokhoba S, Heintz N. Loss of function of axonemal dynein Mdnah5 causes primary ciliary dyskinesia and hydrocephalus. Hum Mol Genet 2002;11:715-21

17. Cremers C, Delleman W. Usher's syndrome, temporal bone pathology. Int J Pediatr Otorhinolaryngol 1988;16:23-30 